\title{
HYDRODYNAMICS CHARACTERISTICS OF THE SMALL SHIPS RUDDERS
}

\author{
Dan Obreja \\ "Dunarea de Jos" University of Galati, \\ Faculty of Naval Architecture, Galati, \\ Domneasca Street, No. 47, 800008, Romania, \\ E-mail:dan.obreja@ugal.ro
}

\begin{abstract}
The estimation of the hydrodynamics characteristics of the small ships rudders is an important issue in the initial design process. The manoeuvring performance depends on the type and geometry of the rudder. The control forces and moments generated by the rudder deflection can be calculated on the basis of the hydrodynamics characteristics of the rudder. A short description of the Mordvinov theoretical model is presented in this paper, in order to determine the hydrodynamics characteristics of a small ship rudder, with the propeller and hull influences. On the basis of the control forces and moments, the optimum position of the rudder stock and the selection of the steering gear can be performed. Using the Mordvinov method, the computer code PHP NM-MAN-HC was developed at the Research Centre of the Naval Architecture Faculty of "Dunarea de Jos" University of Galati. This computer code is integrated in the software platform PHP (Preliminary Hydrodynamics Performance) and is used for didactic applications and practical research activities in the small ship design process.
\end{abstract}

Keywords: small ship, rudder, control forces and moments, computer code.

\section{INTRODUCTION}

The estimation of the manoeuvring performance of the small ships is an important task, starting with the initial design stage. The control forces and moments generated by the rudder deflection depend on the type and geometry of the rudder and can be calculated on the basis of its hydrodynamics characteristics.

A practical method was developed by Mordvinov [1] in order to evaluate the hydrodynamics characteristics of the small ships rudders, with the propeller and hull influences.

On the basis of this method, a computer code was developed at the Research Centre of the Naval Architecture Faculty of "Dunarea de Jos" University of Galati [4]. The computer code was integrated in the software platform PHP (Preliminary Hydrodynamics
Performance), dedicated to the fast investigations of the resistance, powering and manoeuvring performances of the small ships, in the initial design process [3].

Important problems can be solved using the PHP NM-MAN-HC computer code [5]: the estimation of the optimum position of the rudder stock, the selection of the steering gear on the basis of the total torque related to the rudder stock and the calculation of the rudder stock diameter.

A practical demonstration was realised in this paper, to calculate the normal force and the hydrodynamic moment on the rudder stock, depending on the rudder deflection angle, in the case of a small ship having about $15 \mathrm{~m}$ in length. The main dimensions of the small ship are presented in Table 1 . Also, the geometric characteristics of the suspended rudder of the small ship are presented in Table 2. 
Table 1. Main dimensions of the small ship

\begin{tabular}{|l|c|}
\hline \hline \multicolumn{1}{|c|}{ Main characteristics of the small ship } & Value \\
\hline \hline Length of waterline, $\mathrm{L}[\mathrm{m}]$ & 15.1 \\
\hline Breadth, $\mathrm{B}[\mathrm{m}]$ & 3.14 \\
\hline Medium draught, $\mathrm{T}[\mathrm{m}]$ & 0.78 \\
\hline Design speed, $\mathrm{v}[\mathrm{Km} / \mathrm{h}]$ & 20 \\
\hline Block coefficient, $\mathrm{C}_{\mathrm{B}}$ & 0.65 \\
\hline Propeller diameter, $\mathrm{D}_{\mathrm{P}}[\mathrm{m}]$ & 0.6 \\
\hline Propeller thrust, $\mathrm{T}_{\mathrm{p}}[\mathrm{kN}]$ & 6.5 \\
\hline Wake fraction, $\mathrm{w}$ & 0.14 \\
\hline
\end{tabular}

Table 2. Rudder characteristics

\begin{tabular}{|l|c|}
\hline \hline \multicolumn{1}{|c|}{ Main characteristics of the rudder } & Value \\
\hline Hydrodynamic profile & $\begin{array}{c}\text { NACA } \\
0015\end{array}$ \\
\hline \hline Aspect ratio, $\lambda$ & 1.5 \\
\hline Rudder area, $\mathrm{A}_{\mathrm{R}}\left[\mathrm{m}^{2}\right]$ & 0.216 \\
\hline $\begin{array}{l}\text { Rudder area situated in the propeller jet, } \\
\mathrm{A}_{\mathrm{RDp}}\left[\mathrm{m}^{2}\right]\end{array}$ & 0.172 \\
\hline Ballanced area of the rudder, $\mathrm{A}_{\mathrm{C}}\left[\mathrm{m}^{2}\right]$ & 0.063 \\
\hline Chord of the rudder, $\mathrm{b}_{\mathrm{R}}[\mathrm{m}]$ & 0.38 \\
\hline Number of the rudders, $\mathrm{n}_{\mathrm{R}}$ & 1 \\
\hline $\begin{array}{l}\text { Distance between the propeller shaft } \\
\text { and the lower plan of the rudder, } \mathrm{a}_{1}[\mathrm{~m}]\end{array}$ & 0.17 \\
\hline
\end{tabular}

A short description of the Mordvinov method, applied to calculate the hydrodynamics characteristics of the small ships rudders is presented in the next chapter [2].

\section{THEORETICAL METHOD}

The hydrodynamics characteristics of the small ship rudders influence the forces and moments acting on the deflected rudder (Figure 1, [6]).

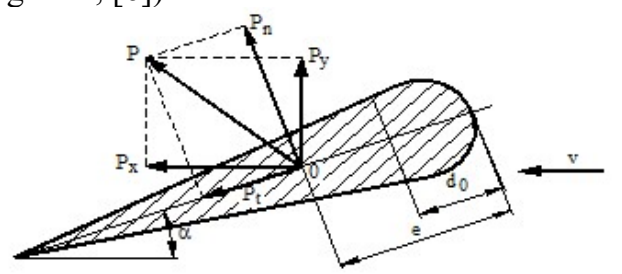

Fig. 1. Rudder forces

For an incident angle $\alpha$, in free stream condition, the following components of the total hydrodynamic force $P$ acting on the deflected rudder can be noted: the drag compo- nent $P_{x}$, the lift component $P_{y}$, the normal force $P_{n}$ and the tangent component $P_{t}$ [6].

The distance of the centre of pressure $\mathrm{O}$ to the leading edge of the rudder is noted with $e$ and $\mathrm{d}_{0}$ is the distance from the rudder stock to the leading edge.

According the Mordvinov method, the normal force $P_{n}$ is determined using the expression

$$
P_{n}=\frac{1}{2} C_{N R H p} \cdot \rho \cdot v_{R}^{2} \cdot A_{R}
$$

where $C_{N R H p}$ is the hydrodynamic coefficient of the normal force, with the influence of the hull and propeller, $\rho$ is the water density and $v_{R}$ is the water speed on the deflected rudder.

Also, the hydrodynamic moment on the rudder stock is calculated on the basis of the following relation

$$
M_{r}=\frac{1}{2} C_{m R H p} \cdot \rho \cdot v_{R}^{2} \cdot A_{R} \cdot b_{R}
$$

where $C_{m R H p}$ is the hydrodynamic coefficient of the moment, with the influence of the hull and propeller, which can be determined using the expression

$$
C_{m R H p}=C_{N R H p} \cdot\left(C_{P R H p}-k_{\delta R}\right)
$$

where $C_{P R H p}$ is the coefficient of the centre of pressure and the balanced area coefficient $k_{\delta R}$ is the ratio

$$
k_{\delta R}=\frac{A_{C}}{A_{R}}
$$

which depends of the rudder stock position.

The water speed on the deflected rudder is calculated using the relations

$$
\begin{gathered}
v_{R}=v \cdot \overline{v_{R}} \\
\overline{v_{R}}=(1-w) \cdot\left(1+A_{R D p} \cdot \frac{C_{T p}}{A_{R}}\right)
\end{gathered}
$$

where $w$ is the wake coefficient and $C_{T p}$ is the propeller loading coefficient.

The detailed calculation of the hydrodynamics coefficients of the normal force and of the moment on the rudder stock is presented in the references [1], [2] and [3].

The values of the mentioned coefficients 
depend on the aspect ratio of the rudder, the chord of the rudder, the relative thickness of the hydrodynamic profile, the balanced area coefficient, the rudder area situated in the propeller jet and the distance between the propeller shaft and the lower plan of the rudder.

Using the described method, the computer code PHP NM-MAN-HC was performed at the Research Centre of the Naval Architecture Faculty of "Dunarea de Jos" University of Galati. Practical applications are presented in the next chapter, in the case of a small ship having about $15 \mathrm{~m}$ in length.

\section{PRACTICAL EVALUATION}

The rudder is the most commonly used passive control device of the ship trajectory in horizontal plane. The course keeping and manoeuvring performances are influenced of the rudder geometry [2].

In the same time, the hydrodynamics forces and moments generated by the rudder deflection angle influence the selection of the steering gear from different data catalogs.

In order to choose the steering gear, the total torque related to the rudder stock must be calculated as the sum of the hydrodynamic moment and the frictional component. Ussualy, the frictional moment is considered as about $20 \%$ from the hydrodynamic moment [7]. As a consequence, the calculation of the hydrodynamic moment related to the rudder stock, depending of the rudder deflection angle is a very important stage in the rudder design process.

A practical evaluation of the rudder hydrodynamics characteristics was performed in the case of a small ship. The main dimensions of the small ship and the rudder characteristics are presented in Table 1 and Table 2 respectively and represent the input data of the mentioned computer code.

The evolution of the normal hydrodynamic force $P_{n}$ depending of the rudder deflection angle $\alpha$ is presented in Figure 2. The curve of the hydrodynamic moment related to the rudder stock $M_{r}$, depending of the rudder deflection angle is shown in Figure 3.

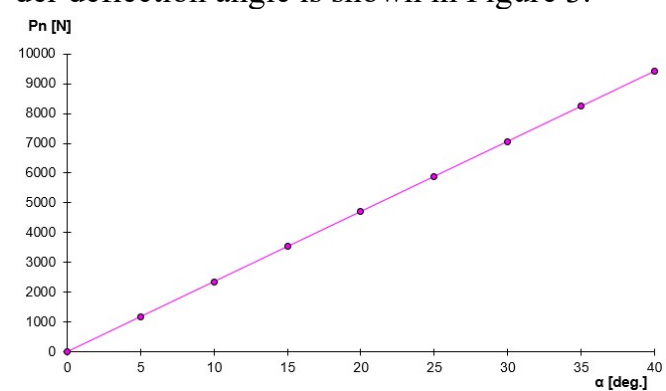

Fig. 2. Normal hydrodynamic force $(\lambda=1.5$; $k_{\delta R}=0.292$; NACA 0015)

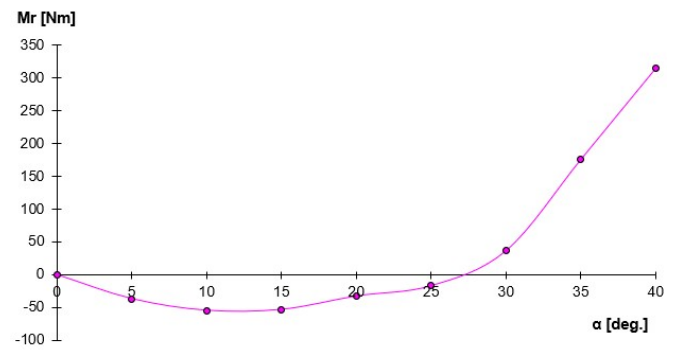

Fig. 3. Hydrodynamic moment related to the rudder stock $\left(\lambda=1.5 ; k_{\delta R}=0.292\right.$; NACA 0015)

The value of the hydrodynamic moment is influenced of the rudder stock position, determined by means of the balanced area coefficient $k_{\delta R}$.

The influence of the rudder stock position on the hydrodynamic moment is presented in Figure 4. Three distinct values of the balanced area coefficient were analized.

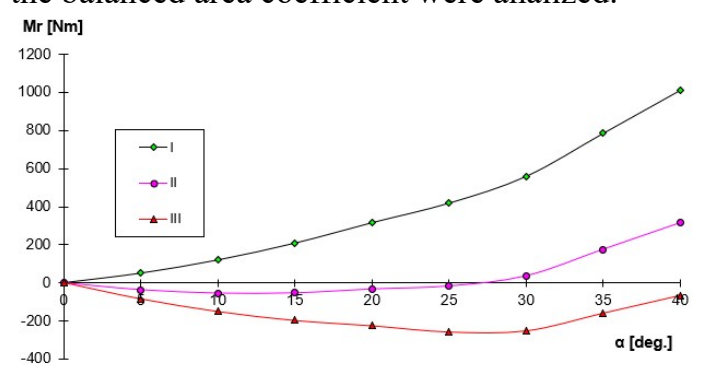

Fig. 4. The influence of the rudder stock position on the hydrodynamic moment $(\lambda=1.5$; NACA 0015; I: $k_{\delta R}=0.097$; II: $k_{\delta R}=0.292$; III: $k_{\delta R}=0.398$ ) 
In the case I (small distance of the rudder stock to the leading edge), the rudder is under-balanced and the hydrodynamic moment related to the rudder stock has only positive values.

In the case II (medium distance of the rudder stock to the leading edge), the rudder is correct-balanced and the hydrodynamic moment related to the rudder stock has both positive and negative values.

In the case III (maximum distance of the rudder stock to the leading edge), the rudder is over-balanced and the hydrodynamic moment related to the rudder stock has only negative values.

The influence of the rudder aspect ratio $\lambda$ on the hydrodynamic moment related to the rudder stock is shown in Figure 5. Three different values were adopted for the rudder aspect ratio.

If the aspect ratio increases, then greater absolute values of the hydrodynamic moment related to the rudder stock can be observed.

In order to obtain a correct-balanced of the rudder, the optimum value of the coefficient $k_{\delta R}$ must be determined, for the selected value of the aspect ratio.

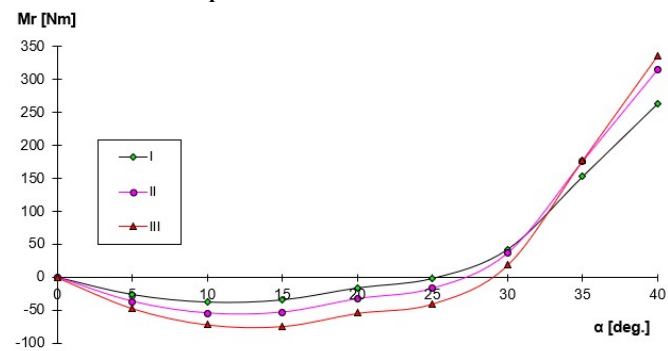

Fig. 5. The influence of the rudder aspect ratio on the hydrodynamic moment $\left(k_{\delta R}=0.292\right.$;

NACA 0015; I: $\lambda=1$; II: $\lambda=1.5$; III: $\lambda=2$ )

The influence of the relative thickness of the rudder profile on the hydrodynamic moment related to the rudder stock is presented in Figure 6. The following types of the hydrodynamics profiles were studied: NACA 0012, NACA 0015 and NACA 0018.

The growth of the relative thickness increase the absolute values of the hydrody- namic moment in the negative domain and decrease the positive values.

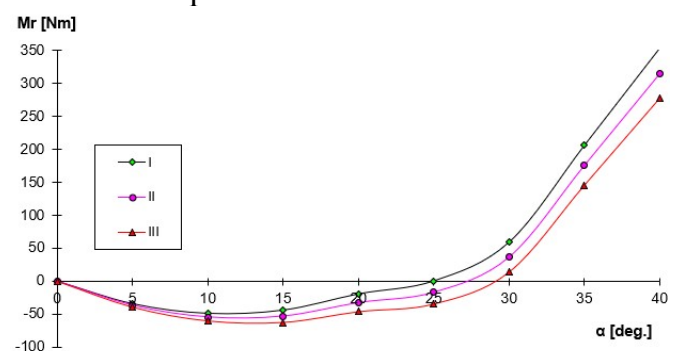

Fig. 6. The influence of the relative thickness of the rudder profile on the hydrodynamic moment $\left(\lambda=1.5 ; k_{\delta R}=0.292\right.$; I: NACA 0012; II: NACA 0015; III: NACA 0018)

The influence of the rudder area situated in the propeller jet $A_{R D p}$ on the hydrodynamic moment related to the rudder stock is shown in Figure 7. Three different values were adopted for the rudder area situated in the propeller jet. The maximum value is equal to the rudder area.

If the rudder area situated in the propeller jet increases, then greater absolute values of the hydrodynamic moment related to the rudder stock can be noted.

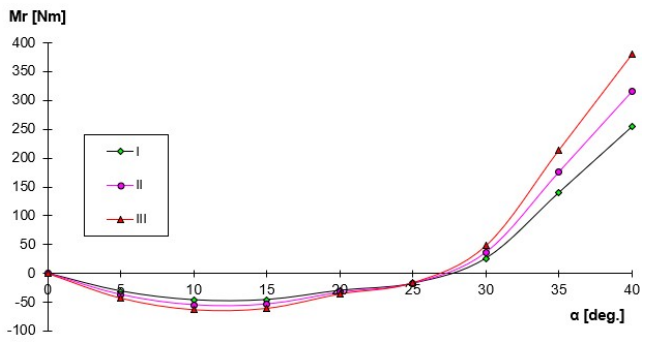

Fig. 7. The influence of the rudder area situated in the propeller jet on the hydrodynamic moment $\left(\lambda=1.5 ; k_{\delta R}=0.292\right.$; NACA 0015; I: $A_{R D p}=0.6 A_{R}$; II: $A_{R D p}=0.8 A_{R}$; III: $A_{R D p}=A_{R}$ )

The influence of the distance $a_{l}$ between the propeller shaft and the lower plan of the rudder, on the hydrodynamic moment related to the rudder stock is presented in Figure 8. Three distinct values were adopted for this distance. The maximum value is half of the propeller diameter. 
Small differences of the hydrodynamic moment were observed in the domain of the lower values of the rudder deflection angle.

If the rudder deflection angle increase, then greater values of the hydrodynamic moment related to the rudder stock can be noted in the case of the minimum value of the distance $a_{l}$.

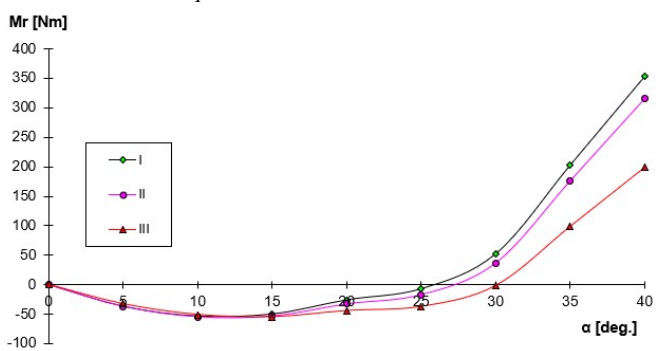

Fig. 8. The influence of the distance between the propeller shaft and the lower plan of the rudder on the hydrodynamic moment

$\left(\lambda=1.5 ; k_{\delta R}=0.292 ;\right.$ NACA $0015 ; \mathrm{I}: \mathrm{a}_{1}=0.1 \mathrm{~m}$; II: $\mathrm{a}_{1}=0.17 \mathrm{~m}$; III: $\mathrm{a}_{1}=0.3 \mathrm{~m}$ )

The capabilities of the computer code analized in this paper can be used in order to perform both didactic applications and research activities related to the rudder design process.

\section{CONCLUDING REMARKS}

The knowledge of the manoeuvring performance represents an important problem of the small ships design.

The control forces and moments determined by the rudder deflection depend on the hydrodynamic characteristics of the rudder.

The rudder geometry influences its hydrodynamic efficiency. In the same time, the influence of the propeller and the hull must be evaluated.

Also, the problem of the structural resistance of the rudder stock must be considered.

In this case, the following objectives related to the rudder stock must be solved:

$\checkmark$ the optimum position; $\checkmark$ the calculation of the total torque (based on the estimation of the hydrodynamic moment);

$\checkmark \quad$ the calculation of the diameter;

$\checkmark \quad$ the selection of the steering gear.

In this paper, a practical method developed by Mordvinov was used, in order to calculate the hydrodynamics characteristics of the small ships rudders, with hull and propeller influences. The theoretical aspects were synthetical described.

A computer code was developed at the Research Centre of the Naval Architecture Faculty of "Dunarea de Jos" University of Galati, in order to solve the mentioned objectives.

The computer code was integrated in the PHP (Preliminary Hydrodynamics Performances) software platform, dedicated to estimate the most important hydrodynamics performances of the small ships (resistance, powering and manoeuvring), starting with the initial design stage.

Practical applications were perform in this paper, using the computer code PHP NM-MAN-HC, in order to investigate the influences of the following input data on the hydrodynamic moment related to the rudder stock:

$\checkmark$ rudder stock position, by means of the balanced area coefficient;

$\checkmark$ rudder aspect ratio;

$\checkmark$ relative thickness of the rudder profile;

$\checkmark$ rudder area situated in the propeller jet;

$\checkmark \quad$ distance between the propeller shaft and the lower plan of the rudder.

The results of practical investigations can be used to adopt the optimum values of the mentioned input data, related to the rudder geometry and the hull-propeller-rudder configuration.

The computer code PHP NM-MAN-HC can be used to develop didactic applications, design activities related to the hydrodynamics characteristics of the small ships rudders 
and applied researches in the manoeuvring domain.

\section{Acknowledgements}

The paper was supported by the Research Centre of the Naval Architecture Faculty of "Dunarea de Jos" University of Galati, which is greatly acknowledged.

The computer code in Java language was realised by George Jagîte, during the student research activities.

\section{REFERENCES}

[1]. Mordvinov, B.G., Spravocinic po malotonajnomu sudostroeniu, Sudostroenie Publishing House, St. Petersburg, 1988 (in Russian).

[2]. Obreja, C.D., "Hydrodynamic particularities of the small ships design", The University Foundation "Dunarea de Jos" Publishing House, Galati, 2004 (in Romanian).
[3]. Obreja, C.D., Pacuraru, S., "Small ships hydrodynamics. Numerical Laboratory", The University Foundation "Dunarea de Jos" Publishing House, Galati, 2016 (in Romanian).

[4]. Obreja, C.D., Jagite, G., "PHP NM-MAN$H C$ ", The Research Centre of the Naval Architecture Faculty in "Dunarea de Jos" University of Galati, 2016.

[5]. Obreja, C.D., "Small ships hydrodynamics software platform", The Annals of "Dunarea de Jos" University of Galati, Fascicle XIShipbuilding, pp. 5-12, 2019.

[6]. Obreja, C.D., "Software platform for manoeuvrability performance estimation in initial ship design", The Annals of "Dunarea de Jos" University of Galati, Fascicle XIShipbuilding, pp. 15-20, 2015.

[7]. Obreja, C.D., Crudu, L., Pacuraru, S. "Ship Manoeuvering", Galati University Press, Galati, 2008 (in Romanian).

Paper received on May $30^{\text {th }}, 2020$ 